\title{
Chromothripsis 18 in multiple myeloma patient with rapid extramedullary relapse
}

Jan Smetana ${ }^{1,2^{*}} \mathbb{D}$, Jan Oppelt ${ }^{3,4}$, Martin Štork ${ }^{5}$, Luděk Pour ${ }^{5}$ and Petr Kuglík ${ }^{1,2}$

\begin{abstract}
Background: Catastrophic chromosomal event known as chromothripsis was proven to be a significant hallmark of poor prognosis in several cancer diseases. While this phenomenon is very rare in among multiple myeloma (MM) patients, its presence in karyotype is associated with very poor prognosis.

Case presentation: In our case, we report a 62 year female patient with rapid progression of multiple myeloma (MM) into extramedullary disease and short overall survival (OS = 23 months). I-FISH investigation revealed presence of gain $1 \mathrm{q} 21$ and hyperdiploidy $(+5,+9,+15)$ in $82 \%$ and $86 \%$, respectively, while IgH rearrangements, del(17)(p13) and del(13)(q14) were evaluated as negative.

Whole-genome profiling using array-CGH showed complex genomic changes including hyperdiploidy $(+3,+5,+9,+11$, $+15,+19)$, monosomy $X$, structural gains (1q21-1q23.1, 1q32-1q44, 16p13.13-16p11.2) and losses (1q23.1-1q32.1; 8p23.

3-8p 11.21) of genetic material and chromothripsis in chromosome 18 with 6 breakpoint areas. Next-generation sequencing showed a total of 338 variants with 1.8\% (6/338) of pathological mutations in NRAS (c.181C > A; p.GIn61Lys) or variants of unknown significance in TP53, CUX1 and POU4F1.
\end{abstract}

Conclusions: Our findings suggest that presence of chromothripsis should be considered as another important genetic hallmark of poor prognosis in MM patients and utilization of genome-wide screening techniques such as arrayCGH and NGS improves the clinical diagnostics of the disease.

Keywords: Multiple myeloma, Chromothripsis, Array-CGH, NGS, Mutation screening

\section{Background}

Multiple myeloma $(\mathrm{MM})$ is characterized by malignant proliferation of clonal plasma cells (PCs) and accumulation of those cells in the bone marrow, formation of osteolytic lesions and presence of monoclonal immunoglobulin in serum and/or urine [1]. It is well established that development of $\mathrm{MM}$ is characterized by clinical and biological heterogeneity driven by proliferation of best adapted clone(s) of PCs, based on Darwin's theory of evolution of species [2]. There are well-known correlations between prognosis and several chromosomal aberrations detected by interphase fluorescence in situ hybridization (I-FISH). Deletion of TP53 in 17p13 loci, translocation $\mathrm{t}(4 ; 14)(\mathrm{p} 16 ; \mathrm{q} 32)$ and gain(1)(q21) detected by I-FISH are

\footnotetext{
* Correspondence: smetty@mail.muni.cz

'Laboratory of Molecular Cytogenetics, Institute of Experimental Biology, Faculty of Science, Masaryk University, Kotlářská 2, 60200 Brno, Czech Republic

2Department of Medical Genetics, University Hospital, Brno, Czech Republic, Černopolní 9, Brno, Czech Republic

Full list of author information is available at the end of the article
}

considered as well established hallmarks of adverse prognosis for MM, both newly diagnosed and relapsed [3, 4]. However, implementation of genome-wide screening techniques, such as array-CGH or recently next generation sequencing (NGS), into clinical practice allows precise description of genetic heterogeneity of cancer diseases, including MM $[5,6]$. In our previous study, we showed that array-CGH in combination with cell sorting is able to detect copy number alterations (CNAs) in $100 \%$ of MM and $65 \%$ of MGUS patients and could be used for monitoring of development of CNAs in clonal populations in MM patients [7, 8]. Other studies also showed that utilization of genome-wide screening techniques allows detection of specific types of genetic lesions with prognostic impact, such as homozygous deletions, loss of heterozygosity (LOH) or chromothripsis in MM [9-12].

The concept of genome chaos was has been recently established a results of extreme stress conditions to cells. Exposure to stress factors leads to complex genomic reorganization, which could ultimately give rise of stable 
genome, however with massive subtypes of chromosome rearrangements known as chromoanasynthesis, chromoanagenesis, chromoplexy or chromothripsis [13, 14]. Chromothripsis $(\mathrm{CTH})$ has been established as a catastrophic event causing complex chromosomal rearrangements involving the shattering of a single chromosome, a small group of chromosomes, or a single chromosome arm [15]. The fragments, or a subset of the fragments, are then stitched together by non-homologous end joining, but in wrong order, place or orientation or also could be missing (copy number loss) or there could be extra copies (copy number gains of DNA) [16]. Due to nature of this phenomenon, it is not surprising that several recent studies showed that incidence of $\mathrm{CTH}$ is associated with extremely poor prognosis in cancer diseases as well as severe phenotype effects in congenital diseases [17-19]. In MM diagnosis, CTH is considered to be a rare chromosomal aberration with incidence of $1 \%$; however, it is associated with a very short overall survival of newly diagnosed patients [20].

In this report, we would like to describe the case of a female MM patient with CTH affecting chromosome 18, hyperdiploid karyotype and pathological mutation in oncogene NRAS and impact on development of the disease. To our knowledge, this is the first published case with CTH 18 and complex genomic screening.

\section{Case presentation}

Female patient without significant comorbidities was diagnosed in 01/2012 at the age of 62 years with symptomatic IgG MM, Durie-Salmon stage III. A, ISS stage 2. At the time of diagnosis, CRAB criteria (hypercalcemia - renal failure - anemia - bone lesions) were met by anemia and bone disease presented by pathological compression of $\mathrm{C} 3$ and L5 vertebrates as detected by MRI.

Treatment of the patient was initiated with 7 cycles of CVD (cyclophosphamid-velcade-dexamethasone). After induction, patient achieved partial remission (PR), and she subsequently underwent in 11/2012 autologous peripheral blood stem cell transplant (PBSCT) with melphalan $\left(200 \mathrm{mg} / \mathrm{m}^{2}\right)$ conditioning. After PBSCT treatment response did not improved and remained PR. Progression of disease occurred after 9 months with incidence of several extramedullary lesions in thoracic vertebrates Th5-7 (08/2013). The patient was then treated by RAD regimen (revlimid-adryamicin-dexamethasone) with supporting radiotherapy. After third cycle, no treatment response was achieved and patient's condition was rapidly worsening. According to extremely aggressive clinical progression, recurrent severe infection complication and overall rapid worsening of the patient's condition, we decided to withdraw oncological treatment and switch to symptomatic-palliative approach. Patient subsequently died due to progression of the disease in $11 / 2013$. Overall survival of the patient was 23 months.

MACS (magnetic-activated cell sorting) technique was used at the time of diagnosis for obtaining enriched $\mathrm{CD} 138^{+}$PCs population for further genetic analyses. In our case, the population purity reached $91 \%$. Detailed cell-sorting protocol was previously by us described elsewhere [21]. Expanded FISH panel for MM patients was used for detection of chromosomal abnormalities with known prognostic impact [22]. We found hyperdiploidy $(+5,+9,+15)$ in $82 \%(82 / 100)$ and gain $1 \mathrm{q} 21$ (CKS1B loci) in $86 \%(86 / 100)$ of scored cells, while $I g H$ rearrangement, $\operatorname{del}(17)(\mathrm{p} 13)$ and $\operatorname{del}(13)(\mathrm{q} 14)$ were evaluated as negative.

Retrospective genome-wide screening showed overall 28 copy number aberrations (CNAs) with the use of microarray-based comparative genomic hybridization (array-CGH). Those aberrations include aneuploidies of odd-numbered chromosomes $(+3,+5,+9,+11,+15,+19)$ and monosomy X. Structural CNAs were observed as deletions in 1q, 8p and 16p, while areas of gain of genetic material were found in $1 \mathrm{q}$ and $16 \mathrm{q}$. Complex chromosomal changes were detected in chromosome 18 and positively identified as chromothripsis (Fig. 1). We observed 6 breakpoint areas, which were accompanied with 12 areas of heterozygous deletions (median of size 2.1 Mbp) and 4 areas of gain of genetic material (median of size 3.3 Mbp). Interestingly, we observed an area of $2.5 \mathrm{Mbp}$ affected with amplification (5-6 copies) in 18q21 (Fig. 2). Moreover, in 16p13.13-16p11.2, we found another area of amplification approximately $22.5 \mathrm{Mb}$ of size, harboring 259 genes spawning from SOCS1 to SLC6A10P. Detailed overview on CNAs is shown in Additional file 1: Table S1.

Mutation screening of 62 leukemia-related genes included in LMA-GeneSGKit ${ }^{\circ}$ (Sistema Genómicos, Spain) showed total of 338 variants. Graphical overview of variant types is shown in Fig. 3. We identified 1.8\% $(6 / 338)$ potentially pathological variants or variants with unknown significance. The most importation mutation was found in NRAS as missense variant in exon 3 (c.181C > A; p.Gln61Lys). Variants with unknown significance were further observed in RAF1, TP53, CUX1 and POU4F1. Detailed information about all variants is available in Additional file 2: Table S2.

\section{Discussion and conclusions}

Identification of chromosomal aberrations in malignant PCs is one of the basic steps in MM staging, which has important impact on disease prognosis of MM disease [23]. FISH investigation with the help of immunostaining (cIg-FISH) or I-FISH performed on enriched CD138 ${ }^{+}$PCs population is still considered as golden standard for cytogenetic testing in MM patients. Metaphase cytogenetics is not suitable technique due to low mitotic activity of 


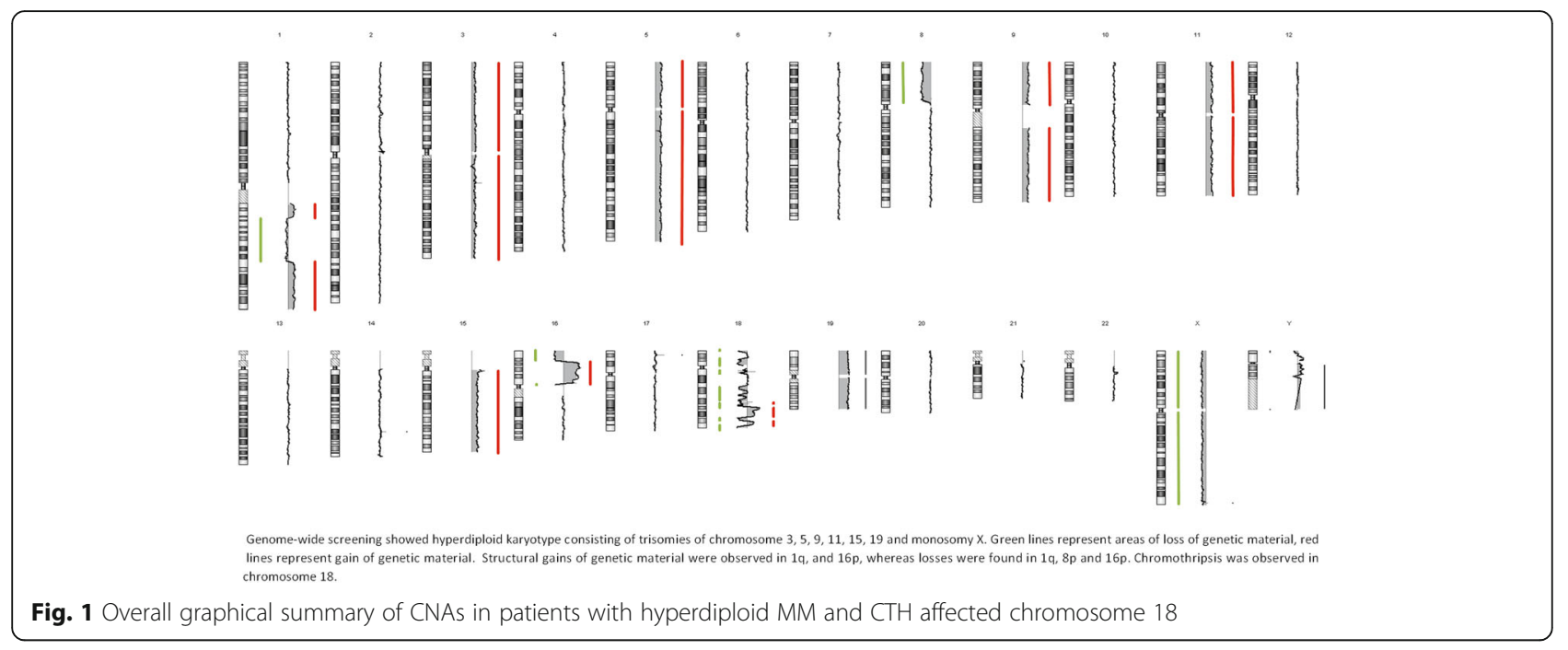

PCs, giving results in $<30 \%$ cases, while I-FISH in combination with cell sorting reaches $90 \%$ of positive investigations [24, 25].

Incidence of $I g H$ translocations, loss of TP53, gain of genetic material in 1q21 area detected by FISH are considered to be negative prognostic factors for both newly diagnosed and relapsed patients [26-28]. While these markers are well established and FISH is considered as gold

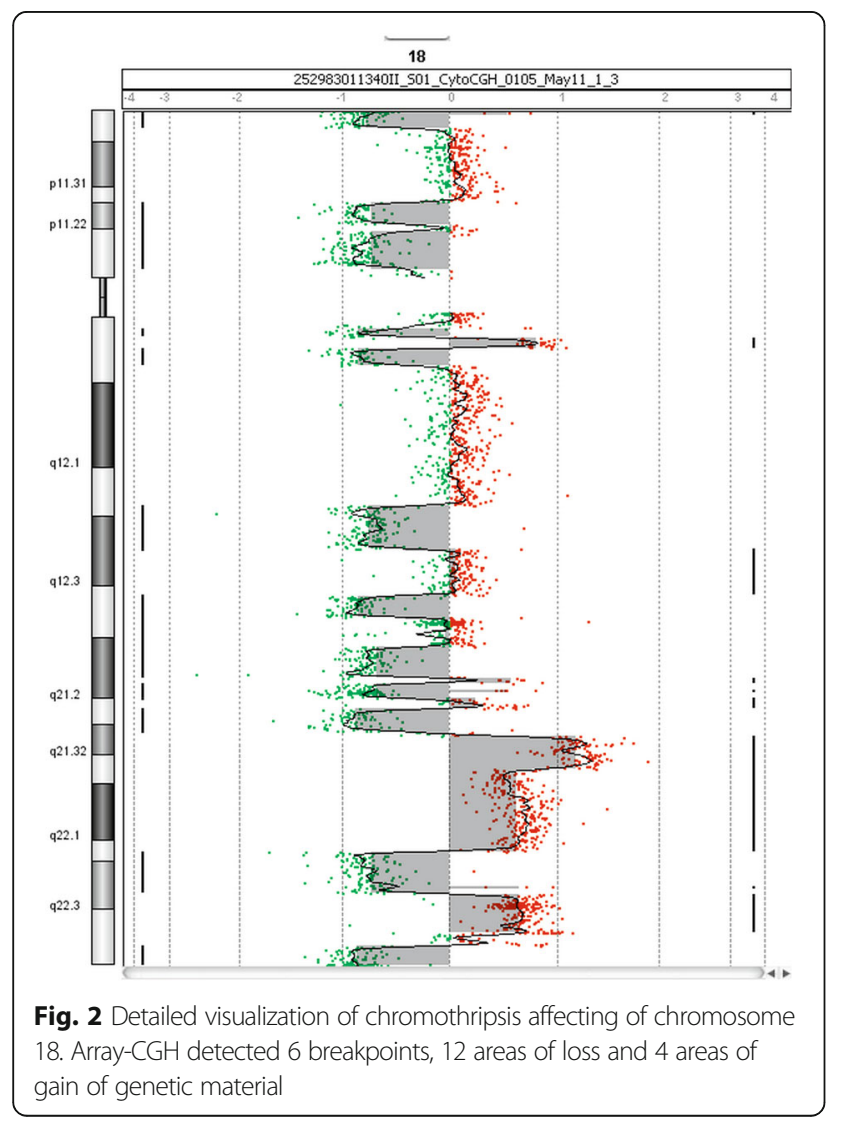

standard of genetic evaluations in $\mathrm{MM}$, the advent of genome-wide screening techniques brought crucial novel information about biology of malignant PCs and genetic basis of the disease, ranging from evaluation of novel CNAs with prognostic impact to discovery of clonal heterogeneity as the principle of development of the disease, sequencing the whole genome of malignant PCs and findings of mutations in critical genes such as $B R A F$, which could be used for personalized treatment protocols $[29,30]$. The utilization in genome-wide profiling in MM patients with the use of array-CGH technique showed that similarly to other hematological malignancies, malignant PCs can harbor a complex chromosomal aberration known as chromothripsis with incidence of $2 \%$ [31]. While the incidence of chromothripsis seems to be rare event in blood cancer diseases, it is only a portion of all types of genomic chaos, which is considered to be one the major contributors to cancer development and progression $[32,33]$. The study of French Myeloma Group also showed that incidence of this catastrophic event detected by microarray techniques in genome of PCs is associated with very poor prognosis and aggressive course of the disease [20]. All these clinical features were present in our patient in a similar manner. This MM case represents a single occurrence of CTH (1.2\%; $1 / 91$ ) in our previously published array-CGH dataset [34]. Overall survival of this patient reached only 23 months, which is in good concordance with data from the French study (OS range from 6 to 32 months, relapse during 10 months from diagnosis). The incidence of chromothripsis affecting chromosome 18 was accompanied with presence of hyperdiploid karyotype, as it is not uncommon in cancer cells [35]. In addition, amplification in 18q affected several genes with known impact in hematological malignancies, such as MALT1 (18q21.32), BCL2 (18q21.33), and KDSR (18q21.33). 


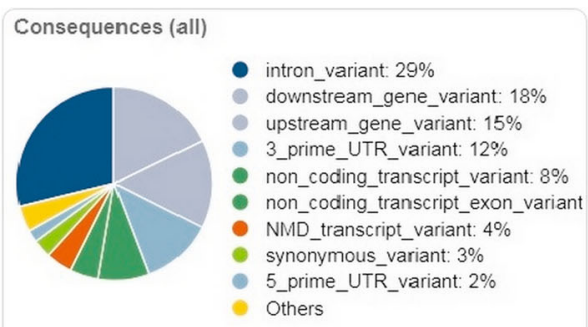

Coding consequences



synonymous variant: $58 \%$

missense variant: $37 \%$

- inframe_deletion: $4 \%$

- frameshift_variant: $1 \%$

- inframe_insertion: $0 \%$

Fig. 3 Overview of 338 variants from next-generation sequencing of 62 hematooncology associated genes from Sistema Genómicos custom panel

While using FISH investigation, when only gain 1q21 was detected as high-impact negative prognostic marker, retrospective utilization of array-CGH showed overall 28 CNAs with areas of amplification of DNA carrying loci of candidate oncogenes in 16p (IL4R, IL21R) and 18q21.32 (PMAIP1). Similar data were previously published with the use of microarray techniques in MM by other groups [36, 37]. Furthermore, losses in 1q and 8p were previously detected by genome-wide profiling in MM patients, however impact of those CNAs on prognosis remains unclear as they were part of complex genomic changes [38, 39].

Benchtop NGS approach together with custom panel of hematonocology-related genes showed possibly harmful variants in $1.8 \%(6 / 338)$. While pathological mutations in NRAS and TP53 were previously described [40, 41], 3'UTR SNP variant:7,668,862 G>A in TP53 was observed in a single patient with Li-Fraumeni syndrome, exon 7 indel CTGTT>C chr3:12,583,765 in $R A F 1$ was found in patient with Noonan syndrome according to Pubmed ICLS (Illumina-Clinical-Services-Laboratory) online public database (https://www.ncbi.nlm.nih.gov/clinvar/submitters/504895). The rest of the variants are annotated in public databases with unknown function, thus their impact is yet to be determined. Of those, intergenic missense variant in CUX1 (rs118010189) and G->C chr13:78,602,199 mutation in POU4F1 promoter flanking region were predicted as intolerant due to possible amino acid substitutions to and with effect of phenotype according to SIFT database [42].

According to aggressive phenotype of this aberration, we suggest to treat $\mathrm{MM}$ patients with $\mathrm{CTH}$ with the aim to reach the best available treatment response [31]. Triplet induction CVD followed by hi-dose chemotherapy and autoPBSCT was best available choice covered by rules of Czech health system at the time of diagnosis. Furthermore, there were no clear data about better efficiency of tandem transplant instead of single transplant 2012. Lenalidomide maintenance was not covered by health insurance at the time of diagnosis. We suggest, that patients with high risk cytogenetic aberrations, like $\mathrm{CTH}$, could profit from lenalidomide maintenance therapy.
Taken together, we can conclude that rapid development and aggressive progression of the disease in our patient was underlined by presence of complex chromosomal aberrations including chromothripsis 18 and amplification in 16p together with causal mutations in several genes associated with hematologic malignancies. While most of found genetic changes have strong association with the development, course and prognosis of the disease, none of them except gain 1q21 are detectable by conventional FISH probe panel used in routine diagnosis. Based on the above-mentioned facts, implementation of genome-wide screening techniques, such as array-CGH, could improve the means of genetic diagnostics in $\mathrm{MM}$ and should be a part of genetic investigation in routine diagnostics in patients with MM.

\section{Additional files}

Additional file 1: Table S1. Aberration list for CNAs detected by array-CGH in MM patient with chromothripsis 18. Output from Agilent Genomic Workbench 7.0.4.0. (XLSX $13 \mathrm{~kb}$ )

Additional file 2: Table S2. Annotation table of 338 called SNP variants from 62 leukemia-associated genes detected NGS Leukemia panel from Sistema Genómicos. (XLSX 91 kb)

\section{Abbreviations}

CGH: comparative genomic hybridization; CNAs: copy number alterations; CTH: chromothripsis; CVD: cyclophosphamid-velcade-dexamethasone; FISH: fluorescence in situ hybridization; LOH: loss of heterozygosity; MGUS: monoclonal gammopathy of undetermined significance; MM: multiple myeloma; NGS: next generation sequencing; PBSCT: autologous peripheral blood stem cell transplant; PCs: plasma cells; PR: partial remission; RAD: revlimid-adryamicin-dexamethasone

\section{Acknowledgments}

We would like to thank Assoc.prof Sabina Ševčíková, PhD for the valuable suggestions and language corrections.

\section{Funding}

This work was supported by internal research funding of Faculty of Science, Masaryk University Brno.

\section{Availability of data and materials}

Additional files are available online in ArrayExpress depository. For peer-reviewing purpose of the database files, please use.

https://www.ebi.ac.uk/arrayexpress/experiments/E-MTAB-6036

\section{Authors' contributions}

Conceived and designed the experiments: JS, JO, PK. Clinical analysis: MS, LP. Cytogenetic and Molecular analysis: JS, JO. Wrote the first draft of the 
manuscript: JS, JO, MS. Made critical revisions and approved final version: JS, JO, MS, PK. All authors read and approved the final manuscript.

\section{Ethics approval and consent to participate}

Present case under submission has been approved by the institutional ethics committee of the University Hospital Brno. This process is in accordance with the Helsinki declaration.

An informed consent was obtained from the patient in the accordance with the requirement of the institutional ethics committee.

\section{Consent for publication}

Informed written consent was obtained from parents for publication of their clinical details and/or clinical images. A copy of the written consent is available for review by the editor of this journal.

\section{Competing interests}

The authors declare that they have no competing interests (financial or non-financial)

\section{Publisher's Note}

Springer Nature remains neutral with regard to jurisdictional claims in published maps and institutional affiliations.

\section{Author details}

'Laboratory of Molecular Cytogenetics, Institute of Experimental Biology, Faculty of Science, Masaryk University, Kotlářská 2, 60200 Brno, Czech Republic. ${ }^{2}$ Department of Medical Genetics, University Hospital, Brno, Czech Republic, Černopolní 9, Brno, Czech Republic. ${ }^{3}$ CEITEC-Central European Institute of Technology, Masaryk University, Kamenice 5, 62500 Brno, Czech Republic. ${ }^{4}$ National Centre for Biomolecular Research, Faculty of Science, Masaryk University, Kotlářská 2, 61137 Brno, Czech Republic. ${ }^{5}$ Department of Internal Medicine-Hematooncology, University Hospital Brno, Jihlavská 20, 62500 Brno, Czech Republic.

\section{Received: 22 November 2017 Accepted: 10 January 2018}

Published online: 18 January 2018

\section{References}

1. Durie $B G$, et al. International uniform response criteria for multiple myeloma. Leukemia. 2006:20(9):1467-73.

2. Morgan GJ, Walker BA, Davies FE. The genetic architecture of multiple myeloma. Nat Rev Cancer. 2012;12(5):335-48.

3. Sonneveld $\mathrm{P}$, et al. Bortezomib-based versus nonbortezomib-based induction treatment before autologous stem-cell transplantation in patients with previously untreated multiple myeloma: a meta-analysis of phase II randomized, controlled trials. J Clin Oncol. 2013;31(26):3279-87.

4. Laubach J, et al. Management of relapsed multiple myeloma: recommendations of the international myeloma working group. Leukemia. 2016;30(5):1005-17.

5. Chapman MA, et al. Initial genome sequencing and analysis of multiple myeloma. Nature. 2011;471(7339):467-72.

6. Egan JB, et al. Whole-genome sequencing of multiple myeloma from diagnosis to plasma cell leukemia reveals genomic initiating events, evolution, and clonal tides. Blood. 2012;120(5):1060-6.

7. Mikulasova A, et al. Genomewide profiling of copy-number alteration in monoclonal gammopathy of undetermined significance. Eur J Haematol. 2016;97(6):568-75.

8. Mikulasova A, et al. Somatic mutation spectrum in monoclonal gammopathy of undetermined significance indicates a less complex genomic landscape compared to multiple myeloma. Haematologica. 2017;

9. Walker BA, et al. A compendium of myeloma-associated chromosomal copy number abnormalities and their prognostic value. Blood. 2010;116(15):e56-65.

10. Walker BA, et al. Integration of global SNP-based mapping and expression arrays reveals key regions, mechanisms, and genes important in the pathogenesis of multiple myeloma. Blood. 2006;108(5):1733-43.

11. Keats JJ, et al. Promiscuous mutations activate the noncanonical NF-kappaB pathway in multiple myeloma. Cancer Cell. 2007;12(2):131-44.

12. Keats JJ, et al. Clonal competition with alternating dominance in multiple myeloma. Blood. 2012;120(5):1067-76.

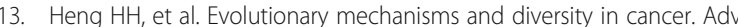
Cancer Res. 2011;112:217-53.
14. Liu G, et al. Genome chaos: survival strategy during crisis. Cell Cycle. 2014;13(4):528-37.

15. Stephens PJ, et al. Massive genomic rearrangement acquired in a single catastrophic event during cancer development. Cell. 2011;144(1):27-40.

16. Kloosterman WP, et al. Constitutional chromothripsis rearrangements involve clustered double-stranded DNA breaks and nonhomologous repair mechanisms. Cell Rep. 2012;1(6):648-55.

17. Skuja $\mathrm{E}$, et al. Chromothripsis and progression-free survival in metastatic colorectal cancer. Mol Clin Oncol. 2017;6(2):182-6.

18. Smida J, et al. Genome-wide analysis of somatic copy number alterations and chromosomal breakages in osteosarcoma. Int J Cancer. 2017;141(4): 816-28.

19. Middelkamp S, et al. Molecular dissection of germline chromothripsis in a developmental context using patient-derived iPS cells. Genome Med. 2017; 9(1):9.

20. Magrangeas F, et al. Chromothripsis identifies a rare and aggressive entity among newly diagnosed multiple myeloma patients. Blood. 2011;118(3): 675-8.

21. Cumova J, et al. Optimization of immunomagnetic selection of myeloma cells from bone marrow using magnetic activated cell sorting. Int J Hematol. 2010:92(2):314-9.

22. Rajan AM, Rajkumar SV. Interpretation of cytogenetic results in multiple myeloma for clinical practice. Blood Cancer J. 2015:5:e365.

23. Palumbo A, et al. Revised international staging system for multiple myeloma: a report from international myeloma working group. J Clin Oncol. 2015;33(26):2863-9.

24. Fonseca $\mathrm{R}$, et al. International myeloma working group molecular classification of multiple myeloma: spotlight review. Leukemia. 2009;23(12):2210-21.

25. Fonseca $\mathrm{R}$, et al. The recurrent lgH translocations are highly associated with nonhyperdiploid variant multiple myeloma. Blood. 2003;102(7):2562-7.

26. Nemec $P$, et al. Gain of $1 q 21$ is an unfavorable genetic prognostic factor for multiple myeloma patients treated with high-dose chemotherapy. Biol Blood Marrow Transplant. 2010;16(4):548-54

27. Greslikova $\mathrm{H}$, et al. Negative prognostic significance of two or more cytogenetic abnormalities in multiple myeloma patients treated with autologous stem cell transplantation. Neoplasma. 2010;57(2):111-7.

28. Smetana J, et al. Gain(1)(q21) is an unfavorable genetic prognostic factor for patients with relapsed multiple myeloma treated with thalidomide but not for those treated with bortezomib. Clin Lymphoma Myeloma Leuk. 2013;13(2):123-30.

29. Lagana A, et al. Integrative network analysis identifies novel drivers of pathogenesis and progression in newly diagnosed multiple myeloma. Leukemia. 2018;32(1):120-30. https://doi.org/10.1038/leu.2017.197.

30. Lohr JG, et al. Widespread genetic heterogeneity in multiple myeloma: implications for targeted therapy. Cancer Cell. 2014;25(1):91-101.

31. Sonneveld $P$, et al. Treatment of multiple myeloma with high-risk cytogenetics: a consensus of the international myeloma working group. Blood. 2016:127(24):2955-62.

32. Poot M. Of simple and complex genome rearrangements, Chromothripsis, Chromoanasynthesis, and chromosome chaos. Mol Syndromol. 2017:8(3): 115-7.

33. Kloosterman WP, Koster J, Molenaar J. Prevalence and clinical implications of chromothripsis in cancer genomes. Curr Opin Oncol. 2014:26(1):64-72.

34. Smetana J, et al. Genome-wide screening of cytogenetic abnormalities in multiple myeloma patients using array-CGH technique: a Czech multicenter experience. Biomed Res Int. 2014;2014:209670.

35. Maciejowski J, de Lange T. Telomeres in cancer: tumour suppression and genome instability. Nat Rev Mol Cell Biol. 2017;18(3):175-86.

36. Bolli N, et al. A DNA target-enrichment approach to detect mutations, copy number changes and immunoglobulin translocations in multiple myeloma. Blood Cancer J. 2016:6(9):e467.

37. Weinhold $\mathrm{N}$, et al. Clonal selection and double-hit events involving tumor suppressor genes underlie relapse in myeloma. Blood. 2016;128(13):1735-44

38. Chng WJ, et al. Correlation between array-comparative genomic hybridization-defined genomic gains and losses and survival: identification of 1p31-32 deletion as a prognostic factor in myeloma. Leukemia. 2010; 24(4):833-42.

39. Walker BA, Morgan GJ. Use of single nucleotide polymorphism-based mapping arrays to detect copy number changes and loss of heterozygosity in multiple myeloma. Clin Lymphoma Myeloma. 2006; 7(3):186-91 
40. Jimenez $\mathrm{C}$, et al. A next-generation sequencing strategy for evaluating the most common genetic abnormalities in multiple myeloma. J Mol Diagn. 2017;19(1):99-106.

41. Kortum KM, et al. Targeted sequencing using a 47 gene multiple myeloma mutation panel (M(3) P) in -17p high risk disease. Br J Haematol. 2015; 168(4):507-10.

42. Ng PC, Henikoff S. Predicting deleterious amino acid substitutions. Genome Res. 2001;11(5):863-74

Submit your next manuscript to BioMed Central and we will help you at every step:

- We accept pre-submission inquiries

- Our selector tool helps you to find the most relevant journal

- We provide round the clock customer support

- Convenient online submission

- Thorough peer review

- Inclusion in PubMed and all major indexing services

- Maximum visibility for your research

Submit your manuscript at www.biomedcentral.com/submit 